\title{
The "Dictionary of the French Academy" digitized, open and free
}

\section{[Le « Dictionnaire de l’Académie française » numérisé, en accès libre et gratuit]}

\author{
Michèle Lenoble-Pinson
}

DOI: 10.18355/XL.2021.14.04.01

\begin{abstract}
Few people are familiar with the Dictionary of the French Academy. However, it provides the official French spelling. Each edition introduces words and graphic modifications. Since 2020, a digital portal provides access to 9 editions, to the conjugation tables of 6,200 verbs and to 900 "Dire, ne pas dire" notices from the French Academy. The rectified forms in 1990 were incorporated at various stages. Hypertext links lead to external resources such as the France Terme database, which offers officially recommended scientific and technical terms, and the Base de données lexicographiques panfrancophone (B.D.L.P.), which brings together lexical varieties from twenty Francophone countries. The Dictionary of the Academy and its supplements, with direct and free access, can be consulted on any digital medium (tablet, mobile phone, computer). www.dictionnaire-academie.fr
\end{abstract}

Key words: Frech Academy, Dictionnary of French Academy,digital portal, spelling

\section{Résumé}

Peu de gens connaissent le Dictionnaire de l'Académie française. Or il fournit l'orthographe française officielle. Chaque édition introduit des mots et des modifications graphiques. Depuis 2020, un portail numérique donne accès aux 9 éditions, aux tableaux de conjugaison de 6200 verbes et à 900 notices « Dire, ne pas dire » de l'Académie française. Les formes rectifiées en 1990 sont intégrées à des stades divers. Des liens hypertextes conduisent à des ressources externes comme la base France Terme, qui propose les termes scientifiques et techniques officiellement recommandés, et la Base de données lexicographiques panfrancophone (B.D.L.P.), qui réunit les variétés lexicales de vingt pays de la Francophonie. Le Dictionnaire de l'Académie et ses compléments, en accès direct et gratuit, sont consultables sur n'importe quel support numérique (tablette, téléphone mobile, ordinateur). www.dictionnaire-academie.fr

Mots-clés: Académie française, Dictionnaire de l’Académie, édition numérique, orthographe

\section{Introduction}

\subsection{Insécurité linguistique des usagers}

Qui connaît le Dictionnaire de l'Académie française? Qui l'utilise ? Peu de personnes, même parmi les très lettrées. Or nombre d'usagers éprouvent un sentiment d'insécurité linguistique avant de prendre la parole en public ou de rédiger un texte. Chacun, en effet, a besoin d'un capital de langue commune, c'est-à-dire des mots bien définis et écrits selon l'orthographe officielle, des expressions correctes et des recommandations grammaticales. Le Dictionnaire de l'Académie française contient un tel capital, dans lequel chacun est invité à puiser en toute sécurité. Encore faut-il que ce dictionnaire soit accessible au plus grand nombre.

XLinguae, Volume 14 Issue 4, October 2021, ISSN 1337-8384, eISSN 2453-711X 


\subsection{Dictionnaire papier et édition numérique}

Depuis 1635, rédiger son dictionnaire constitue la première tâche de l'Académie française. Elle termine la neuvième édition. Pour ceux qui sont attachés au dictionnaire papier sont disponibles les trois premiers volumes, publiés chez Fayard en 1992, 2000 et 2011. Le quatrième et dernier volume est en voie d'achèvement. Toutefois, au $\mathrm{XXI}^{\mathrm{e}}$ siècle, l'édition papier ne suffit plus parce que les techniques informatiques modifient l'accès au dictionnaire. La renumérisation complète de la base informatique du corpus de la neuvième édition a tout changé. Depuis mars 2020, le nouveau portail du Dictionnaire de l'Académie française donne accès gratuitement en ligne à la neuvième édition et à l'intégralité des huit éditions précédentes, toutes numérisées, depuis la première parue en 1694.

\section{Académie française}

Avant d'ouvrir ce grand dictionnaire, il est bon de situer l'Académie française dans l'histoire de la langue. Le latin était la langue exclusive du droit et de son enseignement dans les universités médiévales. Dans les procédures de Toulouse, les juges posaient les questions en français de l'époque, les accusés répondaient en langue d'oc et les verdicts étaient prononcés en latin. Dans la population, les dialectes et les patois dominaient et s'entremêlaient. Le roi réagit.

L'acte officiel de reconnaissance de la langue française comme langue nationale du royaume de France est signé par François I ${ }^{\text {er }}$ le 25 août 1539 au château de VillersCotterêts. C'est l'ordonnance de Villers-Cotterêts. Les articles 110 et 111 installent le français dans les tribunaux et dans l'Administration. Il faut désormais émettre des arrêts clairs et compréhensibles, prononcer, rédiger et publier les registres, enquêtes, contrats, commissions, sentences, testaments et autres actes et exploits de justice « en langage maternel francoys, et non autrement $»$. On peut voir dans cette décision un moyen de protéger les justiciables non lettrés qui ne comprenaient pas le latin et une façon d'écarter les dialectes et les patois au profit de la langue du pouvoir royal.

Le président de la République française Emmanuel Macron l'a annoncé en mars 2018: le château du département de l'Aisne dans lequel François $\mathrm{I}^{\mathrm{er}}$ a signé l'ordonnance relative à la primauté de la langue française sera transformé en Cité de la francophonie. À l'horizon de 2022, le château de Villers-Cotterêts sera entièrement restauré et comprendra un espace ouvert à toutes les cultures francophones.

La langue française se développe à la suite de l'ordonnance de Villers-Cotterêts grâce à l'évolution de l'imprimerie. Infime en 1500, le pourcentage de livres imprimés en français passe à cinquante pour cent en 1575. Du Bellay convainc ses amis poètes de faire chanter la langue française. Le cardinal de Richelieu, ministre du roi Louis XIII, suggère à des écrivains de se réunir dans un cercle inspiré de la Rome antique, une académie, qui favoriserait l'émergence de la belle langue, dont ils seraient les garants. Le 13 mars 1634, rue des Vieilles-Étuves à Paris, s'ouvre la première réunion de l'Académie française. "La principale fonction de l'Académie sera de travailler avec tout le soin et toute la diligence possibles à donner des règles certaines à notre langue et à la rendre pure, éloquente et capable de traiter les arts et les sciences » (article 24). Ainsi Richelieu demande-t-il à l'Académie d'être l'arbitre de la constitution d'une langue commune dans tout le royaume. Il fait entrer dans la Compagnie des auteurs, des magistrats, un avocat, des maréchaux, des archevêques, des Français cultivés, ayant par ailleurs des activités publiques. Ils sont quarante, « les Quarante » (écrit avec une majuscule). Des gens de lettres comme Vaugelas, Boileau, La Fontaine, Corneille, Racine, Bossuet et Fénelon siègent aux côtés d'hommes politiques comme Colbert. 
Si vous demandez à un chauffeur de taxi parisien de vous conduire à l'Académie française - ou même à l'Académie tout court -, il vous y mènera sans poser de question. Chauffeurs de taxi, patrons de bistrot, kiosquiers connaissent l'existence de l'Académie et savent où elle loge, sur la rive gauche de la Seine, dans le $6^{\mathrm{e}}$ arrondissement, quai de Conti, à la hauteur du pont des Arts. Comme la plupart des Parisiens, ils ignorent que cette adresse est celle de l'Institut de France. L'Institut compte cinq classes portant toutes le nom d' «Académie » : l'Académie française (la plus ancienne), l'Académie des inscriptions et des belles-lettres, l'Académie des sciences, l'Académie des beaux-arts, l'Académie des sciences morales et politiques. C'est l'Institut de France qui gère le palais des bords de Seine bâti à l'initiative du cardinal Mazarin.

Le Savoyard Claude Favre, seigneur de Vaugelas, est reçu à l'Académie française parce qu'il s'exprime avec correction. Il n'a encore rien écrit, mais connaît les arcanes subtils de la grammaire, qu'il publiera dans ses Remarques sur la Langue Françoise utiles à tous ceux qui veulent bien parler et bien escrire (1647). Vaugelas devient la cheville ouvrière du Dictionnaire. Il prépare le travail de ses confrères, classe, évalue et choisit les mots. Il définit le bon usage comme « la façon de parler de la plus saine partie de la Cour, conformément à la façon d'écrire de la plus saine partie des auteurs du temps. Quand je dis la Cour, j'y comprends les femmes comme les hommes, et plusieurs personnes de la Ville où le Prince réside, qui par la communication qu'elles ont avec les gens de la Cour participent à sa politesse ». La norme française se fonde sur l'écrit d'une couche sociale dominante.

La légende assure qu'en son dernier instant, Vaugelas faisait encore de la grammaire. « Je m'en vais ou je m'en vas, l'un et l'autre se dit ou se disent. » Cette phrase, il n'a pas pu la prononcer car, dans ses Remarques, il s'élève fermement contre la forme « je vas », pourtant utilisée à la Cour, et lui préfère grammaticalement «je vais », même si cette forme passait pour provinciale et populaire.

\section{Neuf éditions du Dictionnaire de l'Académie française}

La première édition du Dictionnaire de l'Académie paraît en 1694. C'est un dictionnaire de mots courants, sans mots anciens ni termes techniques. En effet, dans son souci de faire un dictionnaire de l'usage contemporain, l'Académie ne répertorie pas les archaïsmes. Quant aux termes techniques, ils sont réunis dans le Dictionnaire des Arts et des Sciences, publié la même année, chez le même éditeur, avec la même présentation, par Thomas Corneille, le frère du dramaturge. C'est un complément indispensable.

Le classement des mots par familles (matrice suivait immédiatement mère), adopté dans la première édition, est abandonné dans les éditions suivantes au profit $\mathrm{du}$ classement alphabétique. Les citations d'auteurs étant exclues par les académiciens, les exemples sont inventés. Au sujet de l'orthographe, « la Compagnie déclare qu'elle désire suivre l'ancienne orthographe qui distingue les gens de lettres d'avec les ignorants et les simples femmes et qu'elle veut la maintenir partout hormis dans les mots où un long et constant usage en aura introduit une contraire " (Mézeray). «L'Académie s'est attachée à l'ancienne orthographe reçue parmi tous les gens de lettres, parce qu'elle aide à faire connaître l'origine des mots. » La graphie contient l'histoire du mot, une mémoire de la langue. L'orthographe française est 
étymologique et conservatrice. Par exemple, l'Académie maintient le $s$ dans beste et teste - qui deviendront bête et tête - alors qu'il n'était plus prononcé.

Quatre éditions du Dictionnaire de l'Académie paraissent au XVIII ${ }^{\mathrm{e}}$ siècle, attestant un important travail sur la langue. La deuxième, rédigée sous la responsabilité de l'abbé Régnier-Desmarais, adopte l'ordre alphabétique et offre une nomenclature enrichie de mots nouveaux et de vocables appartenant au Dictionnaire des Arts et des Sciences. Elle est présentée à Louis XV le 28 juin 1718 .

La troisième édition (1740) introduit des termes d'art et de science entrés dans la langue commune, qui reste néanmoins une langue châtiée, celle de la bonne société. Sous la direction de l'abbé d'Olivet, les académiciens modifient l'orthographe de plus de six mille mots sur les dix-huit mille du Dictionnaire. Ils remplacent le $s$ devant consonne, devenu inutile, par un accent circonflexe (maistre $>$ maître, forest $>$ forêt) et $y$ par $\ddot{i}$ (ayeul > aïeul); ils suppriment les voyelles en hiatus (creu > cru, beuveur $>$ buveur) et diverses consonnes dont on avait encombré les mots par souci étymologique (sçavoir > savoir, faict > fait). Cette édition, en rupture avec la précédente, « instaure en France l'orthographe moderne qui est devenue la nôtre, avec ses défauts et ses qualités » (Nina Catach).

La quatrième édition (1762), parue sous Louis XV, dans sa quarante-septième année de règne, est la dernière publiée sous l'Ancien Régime. Toujours sous la direction de l'abbé d'Olivet sont introduits pas moins de cinq mille mots nouveaux des sciences et des arts. Les simplifications et les régularisations se poursuivent. Beaucoup de lettres grecques sont écartées (déthrôner $>$ détrôner, phanion $>$ fanion). L'accent grave remplace l'accent aigu (mére > mère, fiévre $>$ fièvre). Le $z$ final, marque du pluriel des noms et des participes passés en -é, est remplacé par $s$ (amitiez > amitiés, aimez > aimés).

Pendant la période révolutionnaire, le 8 août 1793, la Convention dissout toutes les Académies de l'Ancien Régime. Le modèle du bon usage passe de la Cour à la Ville, c'est-à-dire aux salons, cafés et clubs, hauts lieux de la vie intellectuelle et politique, il n'en reste pas moins l'usage d'un milieu restreint considéré comme porteur de la norme. Le manuscrit de la cinquième édition du Dictionnaire, préparé par d'Alembert, puis par Marmontel, prêt depuis 1792, est confisqué par la Convention. En 1798, an VII de la République, celle-ci décide de le faire publier en lui adjoignant un supplément parce qu'il est indispensable d'ajouter aux mots de la langue monarchique ceux de la langue de la République. "C'est ce qu'on a fait dans un Appendice. On s'est adressé, pour ce nouveau travail, à des Hommes-de-Lettres, que l'Académie Françoise auroit reçus parmi ses Membres, et que la Révolution a comptés parmi ses partisans les plus éclairés. Ils ne veulent pas être nommés ; leurs noms ne font rien à la chose ; c'est leur travail qu'il faut juger, il est soumis au jugement de la France et de l'Europe » (Discours préliminaire au Dictionnaire de l'Académie Françoise, an VII).

En 1803, quand elle est restaurée, l'Académie désavoue cette édition et son Supplément. Celui-ci contient Les mots nouveaux en usage depuis la Révolution. En douze pages, il regroupe, sous 481 entrées, des néologismes qui relèvent de l'administration (département, grandes écoles), des sciences (aéronaute, aérostier) et de l'actualité révolutionnaire (liberté, égalité [fraternité est absent], liberticide, révolutionnaire, jacobin, terroriste (au sens de "partisan ou agent du système de la Terreur »). S'y trouve aussi le système des poids et mesures : nouvelles unités de monnaie et de mesure, comme franc, mètre, gramme, litre, avec leurs multiples et sous-multiples ainsi que leurs équivalents dans les unités de l'Ancien Régime. Dans 
cette édition du Dictionnaire de l'Académie françoise (sic), la graphie oi est utilisée là où l'on écrit actuellement $a i$, c'est-à-dire dans les terminaisons verbales de l'imparfait de l'indicatif (il devoit, ils dirigeoient) et dans d'autres formes (connoissance, il paroit). Or, à l'époque, l'orthographe dite "de Voltaire » (avec ai) était adoptée depuis longtemps par les écrivains, dont Rivarol. "L'Académie, croyant devoir déférer aveuglément à l'usage » voulait imprimer son nouveau dictionnaire avec cette orthographe, mais les grammairiens comme d'Olivet, Dumarsais, Beauzée et Domergue y étaient opposés. Puisque les Académiciens ne reconnaissent pas la cinquième édition, ils n'intègrent pas les mots du Supplément dans les deux éditions qui paraissent au XIX ${ }^{\mathrm{e}}$ siècle, la sixième (1835) et la septième (1878). Des termes révolutionnaires comme liberticide sont seulement introduits au $\mathrm{XX}^{\mathrm{e}}$ siècle, dans la huitième édition (1932-1935).

$\mathrm{Au} \mathrm{XIX}^{\mathrm{e}}$ siècle, la sixième édition (1835) marque plutôt un retour en arrière. On note néanmoins l'adoption d'importantes modifications en orthographe grammaticale : le rétablissement du $t$ dans les pluriels (enfans > enfants, parens $>$ parents) et l'officialisation de l'orthographe dite «de Voltaire», réforme qu'il avait tant réclamée : le remplacement de oi par ai dans les cas où la diphtongue s'est réduite (anglois > anglais), y compris dans les imparfaits de l'indicatif et les conditionnels.

Élu académicien en 1873, le grand lexicographe Émile Littré est très sollicité, mais il se consacre au Supplément de son Dictionnaire de la langue française et sa présence sous la Coupole est épisodique. Dans la septième édition (1878), les académiciens ajoutent 2500 mots nouveaux. Ils retranchent quelques lettres doubles (consonnance $>$ consonance). Dans les mots tirés du grec, ils suppriment une des lettres étymologiques quand elle ne se prononce pas (phthisie > phtisie, rhythme > rythme). Ils remplacent le tréma par l'accent grave (poëte $>$ poète). L'accent grave prend la place de l'accent aigu dans certains mots (piége $>$ piège, collége $>$ collège). Le trait d'union est supprimé après l'adverbe très et dans beaucoup de mots composés que l'usage a réunis (a-compte > acompte, contre-sens > contresens). L'orthographe académique fait désormais loi, comme le constate Adolphe Hatzfeld en 1893 : «Nos pères respectaient assurément l'Académie française. Mais chacun se croyait autorisé à écrire à sa manière. Il en est tout autrement aujourd'hui. Nul ne se croit plus autorisé à écrire autrement que les autres, c'est-à-dire autrement que le Dictionnaire de l'Académie. On lui demande l'orthographe des mots, comme on demande l'heure à l'Observatoire ».

La huitième édition (1932-1935) comporte des améliorations : définitions revues et corrigées, remaniements d'articles. Devant l'afflux de vocables nouveaux dus aux découvertes et aux inventions (automobile, médecine, aéronautique, électricité, cinéma, etc.), il n'est point aisé de faire le départ entre ceux que l'on accueille (asepsie, bactérie, chronomètre, microbe) dans le Dictionnaire et ceux auxquels on refuse l'entrée, en respectant la règle fondamentale, valable pour tous les temps : «Le bon usage, que recommandait Vaugelas, doit toujours être une règle pour l'Académie, dont la mission n'est pas d'innover, mais de constater et de recueillir les mots admis par les gens qui parlent bien » (Séances du 12 mai et du 21 mai 1885). L'Académie supprime des exemples ainsi que des expressions figurées ou proverbiales sorties de l'usage. Elle remplace l'apostrophe par un trait d'union dans les composés du type grand'mère (> grand-mère) et soude des noms composés (contre-vérité > contrevérité, entr'acte $>$ entracte). Mais ces changements ne sont pas systématiques.

XLinguae, Volume 14 Issue 4, October 2021, ISSN 1337-8384, eISSN 2453-711X 
Pour préparer la neuvième édition, «les quarante Pénélope se remirent à la tapisserie », note le Secrétaire perpétuel Maurice Druon. «Jamais l'humanité n'a eu, en si peu de temps, autant de choses nouvelles à nommer » écrit-il dans la préface. À sa parution en 1992, le premier tome est favorablement accueilli par la critique et par la presse. "Quel ouvrage bénéficie-t-il d'un tel concours de compétences, où le théologien et le prédicateur fameux, le grand philosophe, l'illustre médecin, le savant qui fit progresser la biologie, la plus haute autorité en langue ancienne, le romancier qui fut universitaire, l'historienne d'un quart de la planète, le grand reporter qui a parcouru cette planète en tous sens, l'écrivain qui se souvient d'avoir été diplomate, le critique non seulement des livres mais des mœurs, le mystique qui est aussi pamphlétaire, le juriste qui se cache sous l'essayiste, confrontent et unissent leur expérience et leur savoir afin de conserver son ordre au langage ?» (M. Druon, Discours, 26 mai 1994.)

\section{Avantages des éditions numérisées : https://www.dictionnaire-academie.fr}

Le portail numérique du site de l'Académie française donne accès à la neuvième édition (en voie d'achèvement) du Dictionnaire et aux huit éditions précédentes. Toute recherche relative à un mot de l'extrême fin de l'alphabet est automatiquement et momentanément redirigée vers la huitième édition.

\subsection{Vocabulaire d'hier}

D'une édition à l'autre, les définitions s'améliorent et deviennent plus scientifiques. Le mot écrevisse, longtemps défini par "sorte de petit poisson », devient en 1878 « animal de la classe des crustacés qui vit dans l'eau et qui selon une opinion fausse marche à reculons ». En 2000, «Crustacé décapode vivant dans l'eau douce, dont les pattes antérieures se terminent par des pinces ». - Le mot femme est défini en 1878 par «la femelle, la compagne de l'homme»; en 1932, par «être humain du sexe féminin, la compagne de l'homme »; en 2000, « être humain défini par ses caractères sexuels, qui lui permettent de concevoir et de mettre au monde des enfants ».

Depuis 2020, grâce à la numérisation, les usagers ont accès à l'ensemble de l'entreprise lexicographique commencée par les académiciens en 1634. Selon l'époque du texte ou de l'œuvre à expliquer aux élèves, l'enseignant repère l'édition du Dictionnaire de l'Académie qui y correspond. Rappel : la première date de la fin du XVII ${ }^{\mathrm{e}}$ siècle (1694); quatre éditions paraissent au XVIII ${ }^{\mathrm{e}}$ siècle $(1718,1740,1762$ et 1798); deux au XIX ${ }^{\mathrm{e}}$ siècle (1835 et 1878); une au $\mathrm{XX}^{\mathrm{e}}$ siècle (1932-1935); et la publication de la neuvième commence en 1992.

L'édition numérique du Dictionnaire de l'Académie bénéficie des atouts de la lexicographie de la seconde moitié du $\mathrm{XX}^{\mathrm{e}}$ siècle. Le moteur de recherche permet de circuler dans les neuf éditions en ligne, soit parmi plus de 250000 articles. À partir de la définition d'un mot, en passant d'une édition à l'autre, l'usager peut suivre son évolution sémantique. Deux exemples. Quel était le sens du mot quatorzaine avant qu'il ne désigne, en 2020, le «confinement de quatorze jours imposé aux ressortissants anglais rentrant au Royaume-Uni afin qu'ils évitent de propager la maladie covid $19 »$ ? Le Dictionnaire de l'Académie en ligne montre immédiatement que le terme de droit quatorzaine, attesté dans quatre éditions, de 1762 à 1878, désignait l'« espace de quatorze jours qui s'observait de l'une à l'autre des quatre criées des biens saisis réellement ». Vu son emploi néologique en 2020, quatorzaine entrera-t-il dans la dixième édition avec une définition adaptée ? - Autre exemple. Sous l'Empire, la Grande Armée était formidable. Dans l'édition de 1878, l'adjectif formidable signifie "redoutable, qui est à craindre ou qui inspire une grande crainte »; il vient en effet du latin formidabilis, dérivé de formido, « effroi, terreur ». 
L'armée de Napoléon inspirait de l'effroi aux armées ennemies qui la redoutaient. Le sens du mot passa de la crainte à une appréciation positive de plaisir et d'admiration. Dans ce retournement de sens demeure l'intensité de l'impression. D'abord équivalent d' «effrayant, redoutable », formidable exprime le plaisir, l'étonnement et la qualité supérieure : extra! épatant! super!

L'accès libre et gratuit au portail du Dictionnaire de l'Académie est possible à partir de tout support numérique : ordinateur, tablette, téléphone mobile. Des mises à jour sont faites régulièrement. Évolutif, l'ensemble est conçu pour pouvoir s'enrichir. Le maître artisan des éditions numérisées est Laurent Catach ${ }^{1}$.

\subsection{Vocabulaire d'aujourd'hui}

Pendant le dernier demi-siècle, la langue s'est enrichie d'un nombre considérable de mots. Si l'on compare les deux dernières éditions du Dictionnaire de l'Académie, la huitième, achevée en 1935, et la neuvième, qui est sur le point de l'être, le nombre de mots a doublé. Cet enrichissement lexical est dû moins aux emprunts qu'à l'invention lexicale et à la francophonie. Des mots venus de pays qui partagent l'usage du français, tout en gardant des vocables locaux, se sont ajoutés au patrimoine lexical français. La neuvième édition du Dictionnaire de l'Académie s'accroît de plus de 25000 mots. La nomenclature passe de 32000 (huitième édition) à 60000 mots et le volume d'informations va quasiment doubler à la suite de la description plus détaillée des mots, des emplois et des expressions, de l'introduction de l'étymologie, de la réorganisation en sens numérotés, de la révision de l'orthographe qui tient compte des recommandations de 1990 (voir 4.3) et de l'introduction de titres d'œuvres comme exemples.

À la définition de chaque mot sont adjoints des exemples servant à mettre en évidence les règles syntaxiques et grammaticales qui régissent son emploi et, s'agissant d'un verbe, les constructions justes et la bonne manière de l'accorder. En voici des exemples sous forme d'exercices.

\section{Verbes propres et conjugaison}

Jean a solutionné la difficulté. Non. - Il l'a résolue. Voir résoudre.

Jean clôture le dossier. Non. - Jean clôture son jardin et clôt le dossier. Voir clore.

La mariée est tout émotionnée. Non. - La mariée est tout émue. Voir émouvoir.

Jean sera sanctionné. Non. - La faute sera sanctionnée. Voir sanctionner.

Pour la première fois, la conjugaison des 6200 verbes du Dictionnaire est présentée, sous forme de tableaux, à tous les modes et à tous les temps ainsi qu'aux voix active et passive et à la forme pronominale.

\section{Constructions verbales et prépositives}

Jean se rappelle de la formule. Non. - Il se rappelle la formule. Il se la rappelle. Jean s'en rappelle. Non. - Il s'en souvient. Voir rappeler (se) et souvenir (se).

\footnotetext{
1 Laurent Catach est le fils de Nina Catach, grande dame, spécialiste de l'histoire de l'orthographe française. Voyant l'orthographe comme un outil qui devrait être mis à la portée de tous, elle mena une action militante en faveur d'une orthographe rectifiée. Avec André Goosse, elle fit partie du comité d'experts, dont les travaux aboutirent aux Rectifications de l'orthographe, publiées dans le Journal officiel de la République française (Documents administratifs, 6 décembre 1990).
}

XLinguae, Volume 14 Issue 4, October 2021, ISSN 1337-8384, eISSN 2453-711X 
La société pallie à un défaut. Non. - Elle pallie un défaut. Voir pallier.

On initie une réflexion. Non. - On initie une personne. Voir initier.

Jean travaille sur Paris. Non. - Il pleut sur Paris. Voir sur.

Le bilan est favorable pour la Slovaquie. Non. - Voir favorable.

Croyez-vous à l'Europe ou en l'Europe ? - Voir croire.

\section{Termes propres}

Jean a-t-il commis une faute d'attention ou une faute d'inattention? Voir attention.

Votre expertise, c'est science politique. Non. - Voir expertise.

Au niveau des adjectifs... Non. - Voir niveau.

Est-il arrivé ? Absolument. Non. - Voir oui.

\section{Homonymes et paronymes}

Le fond de la pensée. Le fonds de commerce. Les fonts baptismaux.

Une cession de biens, chez le notaire. La session d'examens, à l'université.

Dans la conjoncture actuelle, nous sommes réduits à certaines conjectures.

Une décennie est une période de dix années, la décade ne compte que dix jours.

\subsection{Neuvième édition et rectifications de l'orthographe de 1990}

Au Moyen Âge, l'orthographe s'est encombrée de lettres inutiles provenant du souci esthétique des copistes (multiplication des $y$ jugés plus jolis que les simples $i$ ) et du désir de rapprocher le français du latin sans se soucier de la phonétique historique ni de l'étymologie, à peu près ignorées alors. Les rectifications de l'orthographe, proposées par le Conseil supérieur de la langue française en 1990 et publiées dans le Journal officiel de la République française du 6 décembre 1990, constituent des variantes orthographiques dont l'usage est recommandé par l'Académie française ${ }^{2}$. De plus en plus d'usagers, qui ne les ont pas apprises, les appliquent spontanément parce que la plupart correspondent à l'évolution naturelle de la prononciation et des graphies. Les deux orthographes, la nouvelle et l'ancienne, sont admises. Aucune des deux ne peut être tenue pour fautive. Le Dictionnaire Hachette (depuis 2005), le Petit Larousse illustré (depuis 2012) et les correcteurs informatiques (Antidote, Cordial) en tiennent compte. Toutes les informations se trouvent sur les deux sites du RENOUVO ou Réseau pour la nouvelle orthographe du français: www.orthographerecommandee.info; www.renouvo.org.

Depuis le premier volume (paru en 1992, nouvelle édition en 2001) de la neuvième édition de son Dictionnaire, l'Académie tient compte des rectifications de 1990, mais pas d'une manière uniforme. Les unes sont enregistrées «à titre définitif»: l'accentuation de certains mots, tels allègement, allègrement, avec leur prononciation habituelle ; cela inclut les futurs et les conditionnels des verbes du type céder: il cèdera, et non plus il cédera; et des mots composés : un essuie-main, des essuiemains; des barmans, etc. Puisque l'on considère l'Académie comme l'autorité en matière d'orthographe, ces formes nouvelles sont appelées à devenir la règle, et les anciennes à devenir désuètes, voire fautives. Les autres rectifications considérées comme permises sont recommandées. Elles sont intégrées de trois façons. Ou bien la graphie nouvelle est la seule indiquée en entrée principale: mémento (accent), photoélectrique (soudure). Ou bien elle fait l'objet d'une variante, en première ou en seconde position : évènement ou événement, moto-cross ou motocross. Ou encore elle

\footnotetext{
${ }^{2}$ M. LENOBLE-PINSON, «L'Académie française et la "nouvelle orthographe" », XLinguae, Volume 12, Issue 1XL, janvier 2019, pp. 3-12. - Cet article donne les sept règles nouvelles et répond aux questions : quelles sont les rectifications ? Combien de mots touchent-elles ? Quelles incohérences et quelles irrégularités suppriment-elles ? Pourquoi les apprenants de français les adoptent-ils volontiers?
} 
fait l'objet d'un symbole, un losange, indiquant qu'une graphie recommandée existe mais n'a pas été retenue dans le Dictionnaire : oignon; les accents circonflexes sur $i$ et sur $u$ : abîme, brûler.

\section{Ressources linguistiques complémentaires}

La version numérique du Dictionnaire de l'Académie présente des avantages par rapport à l'édition papier. Non seulement elle intègre les neuf éditions du Dictionnaire mais, par des liens multiples, elle donne accès à des ressources linguistiques internes ou externes particulièrement riches et intéressantes.

\subsection{Notices de l'Académie « Dire, ne pas dire »}

Les 900 notices mensuelles Dire, ne pas dire, relatives aux difficultés ou aux curiosités de la langue, publiées depuis 2010 sur le site de l'Académie française, sont reliées à environ 1100 entrées du Dictionnaire par 1400 liens hypertextes, qui fonctionnent dans les deux sens. Ces conseils, relatifs aux anglicismes, aux questions de sens, aux faits de syntaxe, intéressent des internautes originaires de plus de cent pays et entraînent un échange fructueux avec le Service du Dictionnaire. Par exemple, dans l'article formidable, lorsque l'usager clique sur la rubrique Dire, ne pas dire, apparaît le conseil : «On évitera de parler d'une famille formidable, d'un endroit formidable, d'une formidable nouveauté, d'une formidable aubaine, et de dire à tout un chacun vous êtes formidable! Il convient de conserver au mot sa valeur: ce qui étonne par sa force, sa puissance, son intensité ou, tout au moins, ce que l'on admire vivement ou approuve chaudement $»$.

\subsection{Base de données France Terme}

Ceux qui se plaisent à truffer leurs paroles et leurs écrits de mots anglo-américains défigurent la langue française sans honorer pour autant celle de Shakespeare. Ce manque de respect à l'égard du français et l'ignorance de son riche patrimoine lexical sont regrettables. De plus, prendre l'habitude d'utiliser un langage sommaire appauvrit l'expression. Tel est le risque qu'entraîne l'emploi du globish (global English), un anglo-américain pauvre et simplifié, réducteur, à la grammaire rudimentaire, qui ne véhicule aucune culture et trahit les idées.

Pourquoi appeler la réunion dans le monde de l'entreprise think-tank et non laboratoire d'idées? Pourquoi pratiquer le brainstorming au lieu du remue-méninges, puis faire un debriefing à la place d'une réunion-bilan ? Ces termes anglo-américains sont inutiles parce que les termes français équivalents existent ; sinon les commissions françaises de terminologie les créent. À Paris, depuis 1996, en effet, ces commissions travaillent pour dire en français, et non en anglais ou en globish, les réalités nouvelles des sciences, des techniques, de l'économie, voire des sciences humaines et sociales, où l'on tendait à adopter simplement le vocabulaire anglais. L'Académie française vérifie la qualité des définitions et des substituts proposés avant que les termes ne soient publiés au J.O. ou Journal officiel de la République française. Les commissions de terminologie contribuent à l'extension du patrimoine lexical que l'on trouve dans les dictionnaires. Toutefois, comme les langues techniques et professionnelles ne ressortissent pas au langage commun, peu de leurs termes rejoignent les pages ou le site du Dictionnaire de l'Académie.

La base de données France Terme, bilingue (français-anglais), installée par la Délégation générale à la langue française et aux langues de France (D.G.L.F.L.F.),

XLinguae, Volume 14 Issue 4, October 2021, ISSN 1337-8384, eISSN 2453-711X 
propose près de neuf mille néologismes et substituts français officialisés en France, destinés à remplacer les emprunts anglo-américains, surtout dans les langues techniques et professionnelles. Dix mille liens hypertextes les relient au Dictionnaire de l'Académie et fonctionnent dans les deux sens.

France Terme est également accessible et consultable gratuitement sur l'internet : www.franceterme.culture.fr.

Domaines: Audiovisuel - Automobile - Biologie - Chimie - Défense Développement durable - Économie et finances - Équipement, transports et tourisme - Informatique - Ingénierie nucléaire - Pétrole et gaz - Santé et médecine - Sciences et techniques spatiales - Sports - Techniques de l'information et de la communication (TIC).

Ainsi, dans France Terme, courriel se substitue à e-mail, la graphie de $C D-R O M$ est francisée en cédérom. Selon le domaine, le verbe coacher est remplacé par accompagner, aider, assister, conseiller, encadrer, entraîner, former, guider, soutenir, suivre... Afin d'éviter le $e$ - (pour électronique) qui sert de préfixe dans $e$ commerce, il est conseillé d'employer la locution en ligne: commerce en ligne, administration en ligne; ou le préfixe télé-, «à distance»: télétravail, téléapprentissage ; ou le préfixe cyber- : cybercafé, cybercriminalité.

Sont recommandés les néologismes : données ouvertes (open data), mégadonnées (big data), dialogue en ligne (chat), féminicide, pornodivulgation et cyberharcèlement (J.O., 07.12.2018). Le système GPS de Géolocalisation Par Satellite est distinct de l'appareil, le géonavigateur.

\subsection{Base de variétés parlées du français dans la Francophonie : B.D.L.P.}

De surcroît, un autre ensemble de plus de cinq mille liens hypertextes rattache le Dictionnaire de l'Académie à la Base de données lexicographiques panfrancophone (B.D.L.P.). Cette base répertorie des variétés du français parlées dans une vingtaine de régions et de pays de la Francophonie. Elle contient plus de 20000 citations extraites de la presse ou de la littérature. Sur 21000 fiches consultables, plus de 2000 se rapportent aux belgicismes: pause-carrière, par exemple, s'emploie pour «congé sabbatique ». En Suisse romande, l'helvétisme fourre à natel désigne la housse pour ordinateur portable. Au Québec, le québécisme pourriel s'applique au courriel indésirable. En Côte d'Ivoire, avoir la bouche sucrée se dit d'un beau parleur ou de celui qui aime flatter. En République démocratique du Congo, l'africanisme eau à ressort désigne l'eau gazeuse et lancer un chameau signifie commettre une faute d'orthographe. - http://www.bdlp.org/

L'accès à la B.D.L.P. constitue une ouverture inédite à la francophonie. En réponse au souhait du président de la République française Emmanuel Macron, on y prépare le Dictionnaire des francophones (D.D.F.), numérique et collaboratif, destiné à montrer, à faire vivre et à partager la richesse de la langue française dans la diversité de ses expressions à travers le monde.

\section{Conclusion : rendez-vous sur le site https://www.dictionnaire-academie.fr.}

Comme toute grande institution, l'Académie française - la Vieille Dame du quai Conti - est mal connue et nombre de clichés masquent son identité et son rôle. En 2020, il importe de donner au Dictionnaire de l'Académie, son œuvre principale, la place dictionnairique qui lui revient. Les neuf éditions numérisées et les compléments sont en accès libre et gratuit sur l'internet. L'interface du site s'adapte aux écrans des ordinateurs, des tablettes et des téléphones mobiles. Ce nouvel outil numérique est riche, souple et performant. 
Depuis la première édition du Dictionnaire parue en 1694, l'internaute peut suivre l'évolution du sens d'un mot au cours des siècles, découvrir des mots disparus et naviguer dans un ensemble de 250000 articles. Le site du Dictionnaire comprend un module de conjugaison de 6200 verbes. Les neuf cents notices mensuelles « Dire, ne pas dire », publiées par l'Académie, sont reliées à plus de mille mots du Dictionnaire. S'ajoutent des liens vers les ressources externes que sont la base France Terme pour la terminologie et la néologie des domaines de spécialités (aéronautique, biologie, économie...) et la Base de données lexicographiques panfrancophone ou B.D.L.P. pour des variantes lexicales du français dans une vingtaine de pays de la Francophonie hors de France.

Le Dictionnaire de l'Académie française est le répertoire « du bon et du bel usage de la langue orale commune, par référence à une norme sociale assez nettement circonscrite, celle de la classe des honnêtes gens » (B. Quemada). Voici un nouveau dictionnaire de référence dans l'espace numérique francophone, au service des internautes qui emploient la langue française ou l'apprennent de par le monde. À terme, la neuvième édition comptera quelque 60000 mots (contre 32000 dans l'édition précédente) et il sera temps de passer à la dixième édition. Le Dictionnaire de l'Académie française, toujours refait, ne s'achèvera pas tant que la langue française sera une langue vivante.

\section{Bibliographic references}

B.D.L.P. Base de données lexicographiques panfrancophone. Available online: http://www.bdlp.org/

CONSEIL SUPÉRIEUR DE LA LANGUE FRANÇAISE. 1990. Les rectifications de l'orthographe. In: Journal officiel de la République française. Documents administratifs, 6 décembre 1990.

DICTIONNAIRE DE L'ACADÉMIE FRANÇAISE. 1992, 2000 et 2011. Paris : Imprimerie nationale - Fayard ; $9^{\mathrm{e}}$ édition, 1992, 2000 et 2011,3 vol., et fascicules parus jusqu'à sérénissime, 19 février 2020. ISBN : 2-11-081249-4. - Éditions numérisées sur le site, Available online: https://www.dictionnaire-academie.fr

FRANCE-TERME. Base terminologique bilingue (français-anglais). Available online: www.franceterme.culture.fr

GREVISSE, M. - GOOSSE, A. 2016. Le bon usage. Louvain-la-Neuve : De Boeck Supérieur ; 16 édition, 1750 p. ISBN : 978-2-8073-0069-9.

GREVISSE, M. - LENOBLE-PINSON, M. 2009. Le français correct. Guide pratique des difficultés. Bruxelles : De Boeck ; 6 e édition, 512 p. ISBN : 978-2-8011-0051-6.

LENOBLE-PINSON, M. 2019. Dire et écrire le droit en français correct. Au plaisir des gens de robe. Bruxelles : Éditions Bruylant; $2^{\mathrm{e}}$ édition, 854 p. ISBN : 978-28027-6047-4.

QUEMADA, B., sous la dir. de, 1997. Les préfaces du Dictionnaire de l'Académie française 1694-1992. Textes, introductions et notes. Paris : Honoré Champion éditeur, ISBN 2-85203-818-8.

RENOUVO. Réseau pour la nouvelle orthographe du français.

Sites : www.orthographe-recommandee.info ; www.renouvo.org

Words: 5959

Characters: 38867 (21,60 standard pages)

XLinguae, Volume 14 Issue 4, October 2021, ISSN 1337-8384, eISSN 2453-711X 
Prof. Michèle Lenoble-Pinson

University Saint-Louis, Brussels

Boulevard du Jardin botanique 43

1000 Brussels

Belgium

michele.lenoble@skynet.be 